\title{
PSICOLOGIA
}

\section{A TERAPIA COGNITIVO-COMPORTAMENTAL NO TRATAMENTO DO TRANSTORNO DE ANSIEDADE GENERALIZADA}

\author{
DOI: http://dx.doi.org/10.31072/rcf.v9i1.557 \\ COGNITIVE-BEHAVIORAL THERAPY IN THE TREATMENT OF GENERALIZED ANXIETY \\ DISORDER
}

Inara Moreno Moura1; Victor Hugo Coelho Rocha²; Gésica Borges Bergamini3; Evelin Samuelsson"; Cristielli Joner ${ }^{5}$; Luiz Fernando Schneider6; Pérsia Regina Menz7.

RESUMO: A ansiedade é uma disfunção emocional que causa sérios prejuízos na vida de um sujeito, e passa a ser reconhecida como patológica quando são exagerados, desproporcionais em relação ao estímulo, ou qualitativamente distintos do que se observa como norma, interferindo assim na qualidade de vida, no conforto emocional ou no desempenho diário do indivíduo. Objetivos: Essa pesquisa tem como objetivo a realização de uma breve revisão bibliográfica a respeito do TAG e das técnicas Cognitiva e Comportamentais mais eficazes no tratamento do TAG, descrever os sintomas da ansiedade generalizada e compreender como as estratégias da Terapia Cognitivo-Comportamental podem ser aplicadas no tratamento psicoterapêutico da ansiedade generalizada. Métodos: Refere-se a um trabalho de revisão bibliográfica, o qual se utilizou de vinte materiais bibliográficos para sua produção. Resultados/Discussão: Pode-se observar que a terapia cognitivo para o TAG, tem grande eficácia, pois oferece ao sujeito uma variabilidade de técnicas que são empregadas de maneira combinadas que causam impacto sobre o transtorno, porque o sujeito passa a vir a preocupação como um processo normal do desenvolvimento humano e não mais como algo patológico, pois o indivíduo é ensinado a reconhecer suas preocupações como um comportamento de aproximação - evitação.

Palavras-chave: Terapia cognitivo-comportamental. Ansiedade. Estratégias cognitivas e comportamentais.

\footnotetext{
1 Psicóloga. Especialista em Terapia Cognitiva Comportamental. Centro Integrado de Aperfeiçoamento Profissional - CIAP. Autora Principal desta pesquisa. E-mail: inaramorenopsi@gmail.com. ORCID: https://orcid.org/0000-0003-2417-2379;

${ }^{2}$ Acadêmico de Psicologia da Faculdade de Educação e Meio Ambiente - FAEMA. Colaborador desta Pesquisa. E-mail: studiovictorocha@gmail.com. ORCID: https://orcid.org/0000-0002-4783-8359;

${ }_{3}$ Mestra, Psicóloga e Orientadora desta pesquisa. Professora da Faculdade de Educação e Meio Ambiente FAEMA. E-mail: gpensemagro@hotmail.com. ORCID: https://orcid.org/0000-0003-0598-5366;

${ }^{4}$ Mestra, Bióloga e Colaboradora desta pesquisa. Professora da Faculdade de Educação e Meio Ambiente FAEMA. E-mail: evelin.samuelsson@faema.edu.br. ORCID: https://orcid.org/0000-0002-0508-2709;

${ }^{5}$ Especialista, Fisioterapeuta e Colaboradora desta pesquisa. Professora da Faculdade de Educação e Meio Ambiente - FAEMA. E-mail: cristielle.joner@faema.edu.br. ORCID: https://orcid.org/0000-0002-7476-667X;

${ }^{6}$ Especialista, Fisioterapeuta e Colaborador desta pesquisa. Professor da Faculdade de Educação e Meio Ambiente - FAEMA. E-mail: luiz.schneider@faema.edu.br. ORCID: https://orcid.org/0000-0002-7945-2581;

${ }^{7}$ Mestra, Fisioterapeuta e Colaboradora desta pesquisa. Professora da Faculdade de Educação e Meio Ambiente -FAEMA. E-mail: persia.menz@faema.edu.br. ORCID: https://orcid.org/0000-0002-1052-6650.
} 
ABSTRACT: Anxiety is an emotional dysfunction that causes serious damage to a subject's life, and is recognized as pathological when exaggerated, disproportionate to the stimulus, or qualitatively different from what is observed as a norm, thus interfering with the quality of life, emotional comfort or the individual's daily performance. Objectives: This research aims at a brief bibliographic review regarding GAD and the most effective Cognitive and Behavioral techniques in the treatment of GAD, to describe the symptoms of generalized anxiety and to understand how the strategies of Cognitive-Behavioral Therapy can be applied in the psychotherapeutic treatment of generalized anxiety. Methods: Refers to a bibliographical review work, which used twenty bibliographic materials for its production. It may be noted that cognitive therapy for GAD has great efficacy because it offers the subject a variability of techniques that are used in a combined manner that impact on the disorder because the subject comes to see the concern as a normal process of human development and no longer as pathological because the individual is taught to recognize their concerns as an approachavoidance behavior.

Keywords: Cognitive-Behavioral Therapy, Anxiety, Cognitive and Behavioral Strategies.

\section{INTRODUÇÃO}

Ansiedade é um sentimento vago e desagradável de medo, apreensão, caracterizado por tensão ou desconforto derivado de antecipação de perigo, de algo desconhecido ou estranho. A ansiedade é uma disfunção emocional que causa sérios prejuízos na vida de um sujeito, e passa a ser reconhecida como patológica quando são exagerados, desproporcionais em relação ao estímulo, ou qualitativamente distintos do que se observa como norma, interferindo assim na qualidade de vida, no conforto emocional ou no desempenho diário do indivíduo.

Indivíduos ansiosos, tendem a superestimar a probabilidade da ocorrência de eventos negativos. Acreditam que são ameaçados pelo perigo e reagem comportando-se segundo essa crença.
Assim erram na sua estimativa de capacidade de lidar como a adversidade e os recursos que têm para tal. Essa subestimação faz com que tirem conclusões incorretas a respeito do ricos real de uma situação.

O Transtorno de Ansiedade Generalizada (TAG) é um transtorno de causas múltiplas. Costuma ser um grande desafio a sua identificação, isso porque é um transtorno com alta taxa de comorbidade. O TAG, tem como principais sintomas a ansiedade e a preocupação excessiva com diversos eventos ou atividade, acontecendo na maior parte dos dias por pelo menos seis meses sendo que o indivíduo considera difícil controlar a preocupação. A ansiedade e a preocupação estão associados a três ou mais dos seguintes sintomas: inquietação, 
fatigabilidade, dificuldade de concentração, irritabilidade, tensão muscular, perturbação do sono.

A terapia Cognitivo-Comportamental é uma terapia de curto prazo, focalizada diretamente no problema. A função cognitiva é fundamental nesse método terapêutico, pois parte do princípio de que o ser humano avalia constantemente a relevância dos acontecimentos, tendo as cognições associadas às reações emocionais. Tem como objetivo a correção das distorções cognitivas, que o indivíduo tem de si mesmo, do mundo e do futuro.

A terapia Cognitivo-Comportamental tem como ideia central o conceito de que as cognições influenciam e controlam as emoções e os comportamentos. Há uma inter-relação entre cognição, emoção e comportamento. Para esse modelo de terapia as psicopatologias são provenientes de perturbações que ocorrem no pensamento de um indivíduo, em que cada indivíduo tem uma percepção específica de interpretar os fatos. Para tanto, busca organizar a perturbação no pensamento que está por trás dos transtornos, identificando assim três níveis de cognições, os pensamentos automáticos, os pressupostos subjacentes e crenças nucleares ou centrais. São a partir do reconhecimento desses conceitos, é que são traçadas as estratégias cognitiva- comportamentais, visando o equilíbrio biopsicossocial do indivíduo.

No TAG os indivíduos caracterizamse pela intensa ansiedade e uma preocupação incontrolável. Considerando essas características do transtorno, o tratamento na TCC, visa à reestruturação cognitiva e a mudança comportamental, proporcionando ao sujeito maior controle $e$ domínio de si, ajudando-o a ver o seu problema sob um novo ângulo.

Essa pesquisa tem como objetivo a realização de uma breve revisão bibliográfica a respeito do TAG e das técnicas Cognitiva e Comportamentais mais eficazes no tratamento do TAG.

\section{MÉTODOS}

Tratou-se de uma pesquisa de revisão bibliográfica cuja trajetória metodológica percorrida foi a de leituras exploratória. Esse é um tipo de pesquisa que segundo Mancini (1) visa à realização de análises e sínteses de informações disponibilizadas por estudos relevantes sobre determinado assunto. Para a concretização do levantamento bibliográfico realizou-se leituras e compreensões da literatura já existente presente em livros do acervo pessoal e também buscas em bases de indexação de resumos de revistas e periódicos eletrônicos consideradas referências na produção de estudos na área da saúde, como Scientific Electronic 
Library Online (SCIELO) e Literatura LatinoAmericana e do Caribe em Ciências da Saúde (LILACS), usando como descritores diversas combinações de palavras chaves como: terapia Cognitivo-Comportamental; ansiedade e estratégias cognitivas e comportamentais. Após a classificação do material bibliográfico, foi realizada a leitura exploratória, obtendo assim uma visão global do material, considerando o interesse ou não à pesquisa. Os estudos selecionados permitiram definir qual material bibliográfico realmente era relevante à pesquisa, sendo então selecionados e citados no transcorrer da pesquisa.

\section{FUNDAMENTAÇÃO TEÓRICA}

\subsection{Ansiedade}

A ansiedade é uma reação natural e imprescindível ao comportamento do ser humano, é um sinal de alerta que permite $o$ indivíduo ficar atento aos perigos iminentes no sentido de que este tome medidas necessárias para lidar com a ameaça, agindo como impulso e motivação a fim de preservá-lo.

Segundo Berrios (apud Nardi) (2): "A palavra ansiedade vem do indo-germânico angh, que indica estreitamento ou constrição". A partir desse termo, originaram-se outros significados em outras línguas, como no grego e latim. No grego "[...] anshein, que significa estrangular, sufocar, oprimir [...]". Em latim “[...] angustus, que exprime desconforto, ango, que significa opressão ou falta de ar, e angere, causar grande constrição, sofrimento, pânico".

Os sintomas da ansiedade podem ser somáticos, somáticos atrasados e motores, como afirma Valle ${ }^{(3)}$. Nos sintomas somáticos podem ser observados, por exemplo, a sudorese, boca seca, respiração curta, frequência cardíaca acelerada, aumento da pressão sanguínea, tensão muscular e dificuldade para respirar, os sintomas somáticos atrasados que podem ser resultados de uma tensão ou estimulação prolongada como a fraqueza muscular, cólica intestinal dores de cabeça e pressão sanguínea elevada.

Os sintomas motores são: inquietação e tamborilar dos dedos das mãos e dos pés e dores musculares. $\mathrm{Na}$ ansiedade há uma intersecção entre os sintomas físicos e psíquicos, onde os sintomas físicos surgem em função das características psicológicas do indivíduo (4,5). Transtornos de ansiedade são atualmente considerados um grande problema para a população mundial, estando associados a elevado sofrimento e limitações, pois além de provocar sofrimento significativo, tais transtornos estão relacionados também a elevados custos além de estar entre os mais debilitantes transtornos psiquiátricos ${ }^{(6)}$. 
Em relação à psicobiologia da ansiedade, Cruz, Zangrossi e Graeff (7), comentam que "Definir uma experiência emocional não é tarefa fácil", e essa tarefa complica mais ainda quando se trata da ansiedade, argumentam também que essa dificuldade pode estar relacionada à questão dos limites de diferenciação da ansiedade normal e da patológica uma vez que, não são claramente identificados, onde em alguns momentos o grau de ansiedade tende a motivar o indivíduo para um melhor desempenho em determinadas tarefas, sendo que, clinicamente o seu diagnóstico será pautado quando houver uma intensidade, durações excessivas, irracional ou quando dissociado de situações ansiogênicas, a partir dessas situações a ansiedade passa a ser claramente um papel desajustador.

Outro ponto que os autores supracitados destacam é a existência da ansiedade como estado ou traço, "[...] A ansiedade-estado é aquela observada em um dado momento vida do indivíduo. [...] A ansiedade-traço é uma característica do indivíduo, ou uma propensão para sentir maior ou menor grau de ansiedade diante de situações ambientais [...]". Reforçando essa ideia, Ferreira et al (8) coloca que "A ansiedade-estado está ligada a um momento ou situação particular [...] causando um estado emocional transitório.
Já a ansiedade-traço está relacionada às características individuais e disposicionais, [...] estando relacionada, diretamente, à personalidade de cada um".

A respeito da predisposição a ansiedade, os autores Castillo et al (9) e Strieder (4) citam que as reações exageradas ao estímulo ansiogênico se desenvolvem, mais comumente, em indivíduos com uma predisposição neurobiológica herdada.

A ansiedade pode causar uma série de transtornos psíquicos. Versiani et al (10), citam que "Os diferentes transtornos de ansiedade caracterizam-se pela presença de sintomas de ansiedade crônicos clinicamente significativos e constituem o grupo mais prevalente dentre os transtornos psiquiátricos".

Por ser um comportamento intrínseco ao ser humano, a ansiedade depende das circunstâncias ou intensidade, por isso D'el Rey et al. (11) destaca a funcionalidade da terapia cognitivo comportamental como será explana adiante. Em níveis normais, trata-se de fenômeno fisiológico responsável pela adaptação do organismo em situações de perigo. No entanto, quando a ansiedade é excedente, em vez de ser útil e contribuir para a adaptação, pode se tornar patológica, prejudicando o funcionamento psíquico e somático desencadeando uma falência da 
capacidade adaptativa, desenvolvendo os transtornos de ansiedade. Quando se trata de diferenciar a ansiedade normal da patológica, deve-se observar a intensidade dos sintomas e também os prejuízos que estes trazem. Para Versiani et al (10) a ansiedade patológica acontece quando "Os pacientes portadores de transtornos de ansiedade apresentam redução significativa da qualidade de vida, com menor produtividade, maior morbidade $\mathrm{e}$ mortalidade, e maiores taxas de comorbidade".

Castillo et al ${ }^{(9)}$ destacam que "[...] A maneira prática de se diferenciar ansiedade normal de ansiedade patológica é basicamente avaliar se a reação ansiosa é de curta duração, autolimitada e relacionada ao estímulo do momento ou não [...]". Ainda sobre a diferenciação da ansiedade normal da patológica, Strieder ${ }^{(4)}$ argumenta que a ansiedade pode ser reconhecida como patológica quando esta além de ser uma reação exagerada e desproporcional ao estímulo "[...] É patológico porque interfere na qualidade de vida, no conforto emocional ou no desempenho diário do indivíduo".

Valle (3), aponta que, quando a ansiedade é excessiva, acontece sem um motivo prévio, ou é desproporcional à situação que a desencadeia, torna-se patológica, gerando alguns transtornos fóbicos e estados de ansiedade.

\subsection{Transtorno de Ansiedade Generalizada - TAG}

Margis e Kapcinski (12) citam que o TAG pode ser entendida como um transtorno multidimensional, com três sistemas de ansiedade: fisiológico, cognitivo e comportamental. Os autores destacam que o TAG consiste na associação da ansiedade e a preocupação excessiva em vários eventos ou atividades, e ocorrem na maioria dos dias por pelo menos seis meses.

Segundo o Manual diagnóstico e estatístico de transtornos mentais (DSM-V 2016), o TAG tem como características principais a ansiedade e a preocupação persistentes e excessivas acerca de vários domínios, incluindo desempenho no trabalho e escolar, que o indivíduo encontra dificuldade em controlar. Além disso, são experimentados sintomas físicos, incluindo inquietação ou sensação de "nervos à flor da pele"; fatigabilidade; dificuldade de concentração ou "ter brancos"; irritabilidade; tensão muscular; e perturbação do sono, embora apenas um sintoma adicional seja exigido para crianças.Os critérios de diagnóstico do TAG no DSM-V (300.02) e na Classificação Internacional das Doenças (CID-10 F.41.1) são: 


\begin{tabular}{|c|c|}
\hline DSM-V - 300.02 & CID-10 - F.41.1 \\
\hline $\begin{array}{l}\text { A. Ansiedade e preocupação excessivas } \\
\text { (expectativa apreensiva), ocorrendo na maioria } \\
\text { dos dias por pelo menos seis meses, com } \\
\text { diversos eventos ou atividades (tais como } \\
\text { desempenho escolar ou profissional). }\end{array}$ & $\begin{array}{l}\text { Ansiedade generalizada e persistente que não } \\
\text { ocorre exclusivamente nem mesmo de modo } \\
\text { preferencial numa situação determinada (a } \\
\text { ansiedade é "flutuante"). } \\
\text { Estado Ansiosa(o) } \\
\text { Neurose Reação de angústia }\end{array}$ \\
\hline $\begin{array}{l}\text { B. O indivíduo considera difícil controlar a } \\
\text { preocupação. }\end{array}$ & $\begin{array}{l}\text { Os sintomas essenciais são variáveis, mas } \\
\text { compreendem nervosismo persistente, } \\
\text { tremores, tensão muscular, transpiração, } \\
\text { sensação de vazio na cabeça, palpitações, } \\
\text { tonturas e desconforto epigástrico. }\end{array}$ \\
\hline $\begin{array}{l}\text { C. A ansiedade e a preocupação estão } \\
\text { associadas com três (ou mais) dos seguintes seis } \\
\text { sintomas (com pelo menos alguns deles } \\
\text { presentes na maioria dos dias nos últimos seis } \\
\text { meses). } \\
\text { Nota: Apenas um item é exigido para crianças. } \\
\text { Inquietação ou sensação de estar com os nervos } \\
\text { à flor da pele. } \\
\text { Fatigabilidade. } \\
\text { Dificuldade em concentrar-se ou sensações de } \\
\text { "branco" na mente. } \\
\text { lrritabilidade. } \\
\text { Tensão muscular. } \\
6 . \text { Perturbação do sono (dificuldade em conciliar } \\
\text { ou manter o sono, ou sono insatisfatório e } \\
\text { inquieto). }\end{array}$ & $\begin{array}{l}\text { Medos de que o paciente ou um de seus } \\
\text { próximos irá brevemente ficar doente ou sofrer } \\
\text { um acidente são frequentemente expressos. }\end{array}$ \\
\hline $\begin{array}{l}\text { D. A ansiedade, a preocupação ou os sintomas } \\
\text { físicos causam sofrimento clinicamente } \\
\text { significativo ou prejuízo no funcionamento social, } \\
\text { profissional ou em outras áreas importantes da } \\
\text { vida do indivíduo. }\end{array}$ & $\begin{array}{l}\text { Exclui: } \\
\text { Neurastenia (F48.0) }\end{array}$ \\
\hline $\begin{array}{l}\text { E. A perturbação não se deve aos efeitos } \\
\text { fisiológicos de uma substância ( } p \text {. ex., droga de } \\
\text { abuso, medicamento) ou a outra condição médica } \\
\text { (p. ex., hipertireoidismo). }\end{array}$ & \\
\hline $\begin{array}{l}\text { F. A perturbação não é mais bem explicada por } \\
\text { outro transtorno mental (p. ex., ansiedade ou } \\
\text { preocupação quanto a ter ataques de pânico no } \\
\text { transtorno de pânico, avaliação negativa no } \\
\text { transtorno de ansiedade social [fobia social], } \\
\text { contaminação ou outras obsessões no transtorno } \\
\text { obsessivo-compulsivo, separação das figuras de } \\
\text { apego no transtorno de ansiedade de separação, } \\
\text { lembranças de eventos traumáticos no transtorno } \\
\text { de estresse pós-traumático, ganho de peso na } \\
\text { anorexia nervosa, queixas físicas no transtorno de } \\
\text { sintomas somáticos, percepção de problemas na } \\
\text { aparência no transtorno dismórfico corporal, ter } \\
\text { uma doença séria no transtorno de ansiedade de } \\
\text { doença ou o conteúdo de crenças delirantes na } \\
\text { esquizofrenia ou transtorno delirante). }\end{array}$ & \\
\hline
\end{tabular}


De acordo com o DSM-V o TAG é um transtorno crônico, que leva o sujeito ao sofrimento e prejuízos significativos. A prevalência é de 12 meses do transtorno de ansiedade generalizada é de $0,9 \%$ entre adolescentes e de 2,9\% entre adultos na comunidade em geral nos Estados Unidos. A prevalência de 12 meses para o transtorno em outros países varia de 0,4 a $3,6 \%$. A manifestação clínica do TAG é relativamente consistente ao longo da vida. A diferença principal entre as faixas etárias está no conteúdo da preocupação do indivíduo. Crianças e adolescentes tendem a se preocupar mais com a escola e o desempenho esportivo, enquanto adultos mais velhos relatam maior preocupação com o bem-estar da família ou da sua própria saúde física.

Assim, o conteúdo da preocupação de um indivíduo tende a ser adequado à idade. Os adultos mais jovens experimentam maior gravidade dos sintomas do que os adultos mais velhos. Muitos indivíduos com transtorno de ansiedade generalizada relatam que se sentem ansiosos e nervosos por toda a vida. A idade média de início do transtorno é 30 anos; entretanto, a idade início se estende por um período muito amplo. O início do transtorno raramente ocorre antes da adolescência.

\subsection{Terapia Cognitivo-Comportamental - TCC}

A TCC é uma terapia de curto prazo, com duração média de cinco a vinte sessões, é focalizada diretamente no problema, para os casos co-mórbidos, o tratamento pode ser estendido por um período acima de vinte sessões. A TCC tem como premissa o princípio de que as cognições influenciam e controlam as emoções e os comportamentos; o modo como o indivíduo age ou se comporta pode afetar de forma significativa os padrões de pensamentos e emoção de um sujeito. A função cognitiva tem um papel essencial na TCC, visto que o ser humano avalia constantemente a relevância dos acontecimentos, tendo as cognições associadas às reações emocionais. Assim, no momento em que ocorre um evento, ocorre à avaliação cognitiva, desencadeando as emoções e consequentemente o comportamento do sujeito ${ }^{(13)}$.

Knapp (14) destaca que a TCC tem como premissa a inter-relação entre cognição, emoção e comportamento. Um evento no cotidiano de um sujeito pode gerar diferentes formas de sentir e agir, mas não é o evento em si que desencadeia as emoções e os comportamentos, e sim o que se pensa em relação ao evento, ou seja, as emoções e os comportamentos são influenciados pelo que o indivíduo pensa.

Observa também as distorções cognitivas, que são alternativas sistemáticas com que os indivíduos interpretam suas experiências. O objetivo da TCC é a correção das distorções 
cognitivas presentes em muitos transtornos psiquiátricos. Por ser a TCC um modelo de terapia de inter-relações não deve ser visto como um método linear, de causa e efeito entre pensamento e resposta emocional, comportamental ou física, mas de um interrelacionamento recíproco entre os pensamentos, sentimentos, comportamentos, fisiologia e ambiente.

Sudak (15) apresenta o modelo cognitivo embasado na conexão entre os pensamentos $e$ as emoções, o comportamento e a fisiologia, em que "Os processos cognitivos - os pensamentos e a avaliação das percepções - afetam os substratos e vias neuras do sistema nervoso central, de modo a produzir estados emocionais e ativar reações fisiológicas e comportamentais".

Coloca ainda que a psicopatologia advém de perturbações que ocorrem no pensamento de um indivíduo, colorindo assim a sua percepção de um modo específico. Durante 0 processo de tratamento a TCC busca organizar a perturbação no pensamento que está por trás dos transtornos, identificando $e$ trabalhando três níveis de cognições, são eles: pensamentos automáticos (PAs); pressupostos subjacentes e crenças nucleares ou centrais. É a partir do conhecimento desses três níveis cognitivos que os terapeutas cognitivo- comportamentais traçam as estratégias buscando o equilíbrio emocional. Esses níveis serão descritos no parágrafo abaixo.

Os PAs ocorrem diariamente, são pensamentos que em sua grande maioria não são percebidos conscientemente, pois além de surgir de forma rápida, involuntária e automática, são praticamente inumeráveis. Os PAs dizem respeito ao self, ao mundo, a outras pessoas e/ou ao futuro. Quando são exagerados, distorcidos, equivocados, irrealistas e disfuncionais possui um importante papel na psicopatologia, pois pode desencadear perturbações muitas vezes, afetivas disfórico. Em muitos casos os PAs são falsos ou apenas parcialmente verdadeiros. Podem ser ativados por eventos externos ou internos. $\mathrm{Na}$ TCC os PAs fazer com que o indivíduo teste a precisão dos mesmos pode ajudar na melhora dos sintomas. Dos níveis de cognição os PAs são os mais fáceis de mudar, uma técnica utilizada para esse fim é sua identificação, seja ela em pensamentos ou imagens. Tem por objetivo ajudar o indivíduo a chegar a conclusões mais evidentes e lógicas.

Os pressupostos subjacentes são as construções disfuncionais subjacentes aos PAs. São compreendidos como regras, padrões, premissas e atitudes de um indivíduo, são desenvolvidas com o decorrer do tempo, levando a expectativas 
em relação a si mesmo e aos outros. São pressupostos condicionais. $\mathrm{O}$ indivíduo acredita que se cumprir certos tipos de regras ou padrões, não haverá problemas, mantendo assim um relacionamento relativamente estável e produtivo. Todavia quando não é cumprido em função de algo, pode tornar o sujeito vulnerável a um transtorno mental, no qual suas crenças nucleares negativas são ativadas.

As crenças centrais são as ideias e conceitos mais profundos, enraizados e fundamentais que a pessoa tem de si. Aqui as crenças são incondicionais, ou seja, o seu pensamento será o mesmo independente das circunstâncias. São aprendidas através das experiências desde a mais tenra idade e se fortalecem ao longo do tempo, se tornando rígidas.

Para os indivíduos em que não há ações corretivas, essas crenças são cristalizadas como verdades absolutas, independentemente de ser ou não o caso. Por ser essa uma crença de cunho rígido, na TCC é o aspecto crucial e fundamental para o processo de cura de um sujeito. Em muitos casos são custosas, danosas e dificultosas de serem alcanças. A correção das crenças nucleares ou centrais é o objetivo maior da TCC. $(14,15)$.

\subsection{Compreendendo as estratégias da} Terapia Cognitivo-Comportamental utilizadas no processo de tratamento da ansiedade generalizada

Integrando técnicas e conceitos vindos de duas principais abordagens, a cognitiva e a comportamental. A TCC focaliza os fatores cognitivos da psicopatologia, reconhecendo a influência do pensamento sobre 0 afeto, 0 comportamento, a biologia e o ambiente.

Shinohara (16) menciona que os objetivos das técnicas cognitivas é identificar os pensamentos automáticos, reconhecer conexões entre estes pensamentos, afetos e comportamentos, testar na realidade e substituir as distorções cognitivas e esquemas disfuncionais por interpretação mais realistas. Enquanto que as técnicas comportamentais envolvem tarefas de observação e experimentação e, são usadas para a modificação de sintomas comportamentais como também para eliciar cognições associadas a comportamentos específicos. Portanto, o objetivo principal do uso dessas técnicas comportamentais na TCC, será o de produzir mudanças nas atitudes do indivíduo e o de testar a validade de suas ideias de competências ou adequação.

Indivíduos ansiosos tendem a superestimar a probabilidade dos eventos negativos ocorrerem, criando assim uma estimativa distorcida de sua capacidade de lidar com a situação. De acordo com Wright; 
Basco; Thase (13) a pessoas que apresentam transtornos de ansiedades, relatam suas experiências subjetivas de medo de forma muito intensa, acompanhadas de sintomas físicos de excitação psíquica, quando apresentadas ao estímulo ameaçador.

As respostas emocionais $\mathrm{e}$ fisiológicas aos estímulos são tão aversivas, e farão de tudo para evitá-las, a fim de que não passe por essas situações novamente. No momento em que as situações são evitadas, há uma recompensa que é o alívio momentâneo, reforçando o seu comportamento evitativo, ou seja, todas as vezes que evita o objeto, situação ou atividade fóbica, 0 comportamento fóbico e suas cognições disfuncionais sobre a situação serão reforçados e seus sintomas serão ainda mais aprofundados.

A TCC se destaca por ser uma das primeiras a dar a atenção ao impacto do pensamento sobre 0 afeto, 0 comportamento, a biologia e o ambiente. Nesse sentido Knapp (14) destaca: “[...] Distorções cognitivas são vieses sistemáticos na forma como indivíduos interpretam suas experiências. [...] $\mathrm{O}$ objetivo da terapia cognitiva é corrigir as distorções do pensamento [...]".

Wright, Basco e Thase ${ }^{(13)}$ descrevem que a TCC é uma abordagem de senso comum, baseada em dois princípios centrais: "[...] 1. nossas cognições têm influência controladora sobre nossas emoções e comportamento; e 2. o modo como agimos ou nos comportamos pode afetar profundamente nossos padrões de pensamentos e nossas emoções [...]". A TCC é utilizada para eliminar e corrigir os padrões de distorções do pensamento, que geram os sintomas a ansiedade produzindo mudanças cognitivo-comportamentais, isso porque, o indivíduo pode trabalhar os dois aspectos de forma simultânea no processo terapêutico.

\subsection{Técnicas Cognitivas}

Comportamentais no tratamento do TAG

Margis e Kapcinski ${ }^{(12)}$ relatam que os indivíduos com TAG tendem a sentir ansiedade intensa e preocupação incontrolável, na intervenção terapêutica devem ser importantes dois aspectos: a preocupação excessiva e incontrolável e a hiperexcitabilidade persistente, que consiste em manifestações físicas incontroláveis pertinentes à tensão.

Os autores supracitados, argumentam que os sujeitos com TAG tem uma percepção elevada de perigo ou ameaça; a superestimação do acontecimento do perigo está associada a uma percepção de baixa capacidade de lidar com essa ameaça. A apreensão em relação ao futuro, ao que ainda não aconteceu é constante no 
TAG, logo a interpretação constante negativa dos estímulos, dos eventos ou das situações é decisiva para o entendimento dos transtornos de ansiedade.

Segundo Knapp ${ }^{(14)}$ a reestruturação cognitiva é o foco constante do trabalho conjunto do terapeuta e do paciente. $O$ objetivo aqui é o de ajudar o paciente a identificar os pensamentos disfuncionais e avaliar outros pensamentos mais assertivos como alternativa. Nesse sentido, a identificação e 0 questionamento possibilitará que os pensamentos pertinentes à ansiedade excessiva sejam desaprendidos e cognições mais realista e apropriadas sejam consideradas. A contestação das cognições ansiogênicas envolve outros aspectos, por exemplo: a consideração de que os pensamentos são hipótese, e precisam ser baseados em evidências, que podem ser confirmadas ou descartadas; fundamentar-se nas evidências para examinar a validade da crença; e gerar predições plausíveis do evento a partir da análise da situação.

Wright, Basco e Thase ${ }^{(13)}$ consideram que as sequências de intervenções são semelhantes para 0 tratamento para ansiedade pode ser usada em outros transtornos ansiosos. Incialmente são avaliados os sintomas e os gatilhos que geram ansiedades, verificando quais as estratégias de enfrentamento existente. Em seguida, são definidos os alvos específicos de intervenções que irão direcionar o curso da terapia, ensinando habilidades básicas para o enfrentamento dos pensamentos, sentimentos e comportamentos que caracterizam a ansiedade e por fim essas habilidades serão utilizadas para auxiliar o indivíduo em situações que geram ansiedade.

A Terapia Cognitiva Comportamental em Grupo - TCCG, constitui-se numa modalidade de terapia que vem sendo cada vez mais utilizada no tratamento de vários transtornos psiquiátricos, devido ser comprovado a eficácia da teoria e técnica, além do mais, reduzir o custo e possibilitar o acesso a um número consideravelmente maior de pacientes, logo, o atendimento em grupo tem sido utilizado e visto sua funcionalidade (17).

\section{A reestruturação cognitiva, o} manejo da ansiedade e a preocupação excessiva, são as principais técnicas da TCC, que podem ser usadas especificamente no TAG e também em outros transtornos ansiosos. Serão descritas de acordo com as ideias de Knapp (14); Manfro, Heldt e Shinohara (18); Margis e Kapcinski (12); Sudak (15); Shinohara ${ }^{(16)}$ e Wright, Basco e Thase ${ }^{(13)}$.

$\mathrm{Na}$ reestruturação cognitiva são trabalhadas 0 registro de pensamentos disfuncional (RPD), a descoberta guiada, o 
questionamento socrático e a descastratofização.

O RPD é utilizado para auxiliar no rastreamento dos pensamentos que foram acionados pela situação estimuladora a que geraram a emoção e o comportamento seguinte. É um exercício que visa a capacitação dos pacientes a descobrir, esclarecer e alterar os significados que atribuíram a eventos perturbadores e compor uma resposta alternativas ou racional.

A descoberta guiada é realizada por meio do questionamento socrático, é uma das pedras angulares da terapia cognitiva. Tem por objetivo guiar o sujeito a um pensamento mais consciente permitindo que o mesmo tenha uma compreensão maior a respeito do seu pensamento distorcido.

Na descatastrofização, o paciente é levado a avaliar as consequências, que são manejáveis, suportáveis e limitadas no tempo. Aqui é trabalhado alguns procedimentos que podem ajudar na redução das previsões catastróficas; são eles: fazer uma estimativa da probabilidade de ocorrer um resultado catastrófico, avaliando as evidências a favor e contra a probabilidade de acontecer o evento; revisar a lista de evidências, avaliando a percepção de controle e criar-se um plano de ação, desenvolvendo um plano de enfrentamento caso a catástrofe ocorra, reavaliar a percepção da probabilidade do resultado catastrófico, fazendo uma análise com o paciente e questionando como foi falar sobre seus pensamentos catastróficos. Deve-se haver um reforçamento sobre 0 valor $\mathrm{da}$ descatastrofização como parte do tratamento terapêutico.

O manejo da ansiedade é realizado por meio do treinamento de relaxamento, higiene do sono e manejo do tempo.

O relaxamento objetiva o alívio dos sintomas ligados ao componente fisiológico da ansiedade visando a interrupção a associação aprendida entre a hiperexcitabilidade e a preocupação. A respiração de um sujeito ansioso tende a ser superficial, acelerada, ofegante, alternando tentativas de retenção do ar com a inspiração de grande volume de ar. Aqui o foco do relaxamento é auxiliar os pacientes a aprenderem a alcançar uma resposta de relaxamento - um estado de calma mental e física. O relaxamento pode ser diafragmático, muscular, visual e meditativo. Todos os relaxamentos visam a liberação da tensão, para que o corpo relaxe e os sentimentos ansiógenos reduzam.

$\mathrm{Na}$ higiene do sono os sujeitos são orientados a realizar exercícios físicos exclusivamente durante a manhã ou nas 
primeiras horas da tarde; comer uma refeição leve acompanhada de ingestão de água limitada durante o jantar; e ainda evitar o uso da nicotina, do álcool e de bebidas que contenham cafeína. Regularizar o horário de deitar e levantar, usar o quarto somente quando for dormir e manter atividade sexual.

O manejo do tempo, objetiva a redução da ansiedade diária, visto que o sujeito poderá estabelecer suas atividades prioritárias mantendo assim a sensação de domínio em relação ao seu dia-a-dia e seus compromissos.

$\mathrm{Na}$ preocupação excessiva as técnicas principais são: exposição à preocupação, a designação de um tempo para preocupação, parada de pensamentos e distração.

A exposição à preocupação baseiase em estudos que demonstraram o papel da preocupação na manutenção da TAG. Margis e Kapcinski (12) citam 5 passos do modelo proposto por Carske e colaboradores para trabalhar a exposição à preocupação, são eles: 1. Identificar e registrar as esferas de maior preocupação do cliente e ordená-las hierarquicamente; 2. Treinar a imaginação com cenas agradáveis e com cenas que causem desconforto e preocupação (netas, considerar o pior desfecho possível). Quando esta fase estiver acontecendo com nitidez, passa-se para próxima; 3 . Escolher a preocupação menos perturbadora do registro inicial e praticar o treinamento de imaginação: evocando a preocupação por meio da concentração do cliente em seus pensamentos ansioso e na imaginação do pior resultado que teme; 4. Assim que o cliente completar a fase 3 , o terapeuta deve estimular a rememoração desses pensamentos e imagens por cerca de 25 a 30 minutos; 5 . Gerar alternativas para os resultados temidos e propostos nos itens anteriores.

\section{A técnica de designar um tempo} para preocupar-se visa auxiliar o sujeito na redução da associação entre preocupação e indicativos de ansiedade, portanto, diminuir a intensidade e a frequência das respostas d preocupação, propicia ao paciente o desenvolvimento de um senso de controle quanto a sua preocupação. O paciente é orientado a escolher um intervalo de tempo, esse período de tempo deve ocorrer todos os dias no mesmo local e horário e não pode ser associado ao relaxamento ou ao trabalho. Assim o sujeito, deve postergar sua preocupação para esse local e horário estabelecido.

A parada de pensamento consiste na interrupção dos pensamentos negativos, substituindo-os por pensamentos adaptativos e mais positivos. É um 
procedimento que visa ao reconhecimento de que está ativo um processo de pensamento disfuncional, dar um auto comando para interromper os pensamentos, e evocar uma imagem visual reforçando o comando mudando a imagem para uma cena agradável ou relaxante

A distração consiste em solicitar ao paciente que se concentre de forma intensa em imagens mentais positivas e calmantes ou em algum objeto externo. Desse modo, a técnica da distração apresenta-se mais eficaz, isso porque os pensamentos guiados pela ansiedade são atenuados.

\section{CONSIDERAÇÕES FINAIS}

A ansiedade é possivelmente a disfunção emocional que mais atinge a qualidade de vida de um indivíduo, e pode desencadear uma série de prejuízos nas áreas do funcionamento social, acadêmicos e ocupacional. O TAG é um transtorno complexo, com uma grande variedade de sintomas para os quais existem diferentes técnicas na TCC.

A TCC é uma terapia de curto prazo, com duração média de cinco a vinte sessões, é focada diretamente no problema, para os casos co-mórbidos, o tratamento pode ser estendido por um período acima de vinte sessões.

A TCC tem se mostrado efetiva no TAG e, que surge como uma alternativa ao uso de medicamentos, uma vez que é mais tolerada pelos pacientes por não ter efeitos colaterais, e também por apresentar significativo custo-benefício.

A TCC é descrita, fundamentalmente, na conexão entre os pensamentos e as emoções, o comportamento e a fisiologia. Identifica e trabalha três níveis de cognições, são eles: pensamentos automáticos $\quad(\mathrm{PA})$; pressupostos subjacentes e crenças nucleares ou centrais. É a partir do conhecimento desses três níveis cognitivos, que os terapeutas cognitivo-comportamentais traçam as estratégias buscando 0 equilíbrio emocional.

Partindo dos princípios da TCC de que as cognições influenciam e controlam as emoções e comportamentos e que o modo como o indivíduo age ou se comporta pode afetar de forma significativa os padrões de pensamentos e emoção de um sujeito, faz com que a função cognitiva tenha um papel essencial no tratamento do TAG. O foco da TCC nesse transtorno é a reestruturação cognitiva, que visa a identificação dos pensamentos disfuncionais e ensina 0 sujeito a considerar outros pensamentos mais assertivos como alternativa. A partir da identificação e também dos questionamentos dos pensamentos disfuncionais, há a possibilidade de que esses pensamentos sejam desaprendidos 
e as cognições mais realistas passam a ser consideradas.

Estudos apontam que após seguimento de seis meses na TCC, a taxa de recuperação chega a $51 \%$ no tratamento do TAG. Atualmente, o modelo CognitivoComportamental, é o mais prevalente e o que obtém melhores respostas ao tratamento do TAG isso porque incide necessariamente em provocar mudanças na forma alterada de perceber e raciocinar acerca do ambiente e especificamente sobre aquilo que gera a ansiedade (terapia cognitiva) e também por promover mudanças no comportamento do sujeito ansioso (terapia comportamental). A eficácia desse método pode ser duradoura, pois o uso das técnicas que permitem tanto a extinção do medo condicionado quanto da regulação cognitiva de emoções para o ansioso, prevenindo assim a recaída.

A técnica de Mindfulness tem sido considerada no tratamento do TAG. Mindfulness é uma forma específica de atenção plena, concentração no momento atual, intencional, e sem julgamento. Significa estar plenamente em contato com a vivência do momento, sem estar submergido por ela.

O Mindfulness apresenta-se como uma nova perspectiva da prática da terapia Cognitivo-Comportamental no tratamento do TAG.
A terapia Cognitivo-Comportamental baseada em mindfulness e aceitação é uma nova abordagem da psicologia, que incorporou à terapia CognitivoComportamental conceitos provenientes das tradições espirituais orientais. Esta abordagem terapêutica tem como principal finalidade, ajudar o cliente a identificar e vivenciar de forma consciente suas emoções sem ficar dominado por elas. Combinar a TCC tradicional à mindfulness e aceitação no TAG é um desafio que pode originar resultados positivos, visto que a aceitação da ansiedade facilita 0 seu manejo podendo colaborar para potencializar e facilitar a reestruturação cognitiva.

A ansiedade vem se configurando como um dos principais problemas da atualidade, que pode estar sendo intensificado pelos avanços tecnológicos desenfreados, pressões sociais e também econômicas.

A ansiedade e a preocupação somado a inquietação, fatigabilidade, dificuldade de concentração, irritabilidade, tensão muscular, perturbação do sono são os sintomas principais sintomas do TAG.

A terapia Cognitivo-Comportamental é ultimamente uma das abordagens que mais cresce na psicologia, configurando-se, apesar de sua origem recente, numa das mais procuradas do mundo para o 
tratamento da TAG, isso porque utiliza técnicas cognitivas e comportamentais.

Assim, as técnicas terapêuticas dessa abordagem visam identificar e testar cognições distorcidas dos pacientes, guiando-os para a construção de esquemas cognitivos mais funcionais a sua realidade (19).

No que se refere aos passos iniciais de manuseio com 0 transtorno de ansiedade generalizada, os terapeutas devem realizar uma apresentação do modelo cognitivo do TAG e da TCC, além de uma minuciosa investigação acerca de atestar ou não a hipótese diagnóstica de
TAG, além de uma fiel conceitualização de caso ${ }^{(20)}$.

Diante da conclusão desse estudo, pode-se observar que a terapia cognitiva para o TAG, tem grande eficácia, pois oferece ao sujeito uma variabilidade de técnicas que são empregadas de maneira combinadas que causam impacto sobre o transtorno, porque o sujeito passa a vir a preocupação como um processo normal do desenvolvimento humano e não mais como algo patológico, pois o indivíduo é ensinado a reconhecer suas preocupações como um comportamento de aproximação - evitação.

\section{REFERÊNCIAS}

1. Mancini MC, Sampaio RF. Quando o Objeto de Estudo é a Literatura: Estudos de Revisão. Rev. Brasileira de Fisioterapia 2006; 10(4): 361-472. [citado em 20 de agosto de 2017]. Disponível em: http://www.scielo.br/pdf/rbfis/v10n4/00.pdf.

2. Nardi EA. História. In: Nardi EA, Valença MA. Transtorno de Pânico: Diagnóstico e Tratamento. Rio de Janeiro: Guanabara Koogan; 2005. p. 01-21.

3. Valle LG. Transtorno de Pânico. In: Abreu CN, Roso M, organizadores. Psicoterapias Cognitiva e Construtivista: Novas Fronteiras da Prática Clínica. Porto Alegre (RS): Artmed; 2003. p. 181-193.

4. Strieder R. Depressão e Ansiedade em Profissionais da Educação das Regiões da Amerios e da AMEOSC. Rev Roteiro 2009; 34(2): 243-268. [citado em 20 de agosto de 2017].

Disponível

em: http://editora.unoesc.edu.br/index.php/rotei ro/article/view/307/60.

5. Zuardi AW. Características Básicas do Transtorno de Ansiedade Generalizada. Rev Medicina Ribeirão Preto Online 2017; 50(Supl.1): 51-55. [citado em 15 setembro 2017]. Disponível em: https://www.journals.usp.br/rmrp/article/vie w/127538/124632.

6. Sousa LPC, Vedana KGG, Miasso Al. Adesão ao Tratamento Medicamentoso por Pessoas com Transtorno de Ansiedade. Rev Cogitare Enfermagem 2016; 21(1): 111. [citado em 15 setembro 2017]. Disponível em: http://revistas.ufpr.br/cogitare/article/view/4 3510/27515.

7. Cruz APM, Zangrossi JH, Graeff FG. Psicobiologia da Ansiedade. In: Rangé B. (org.). Psicoterapia Comportamental e Cognitiva: Pesquisa, Prática, Aplicações e Problemas. Campinas (SP): Psy; 1998. 
8. Ferreira $\mathrm{CL}$, Almondes KM, Braga LP, Mata ANS, Lemos CA, Maia EMC. Universidade, Contexto Ansiogênico? Avaliação de Traço e Estado de Ansiedade em Estudantes do Ciclo Básico. Rev Ciência \& Saúde Coletiva 2009; 14(3): 973981. [citado em 20 de agosto de 2017]. Disponível em: https://www.researchgate.net/profile/Eulali a_Maia/publication/26312294_Evaluation of_trait_and_state_anxiety_in_first_year_st udents/links/55f2d2 d508ae6a34f65de4f2.p df.

9. Castillo ARGL, Recondo R, Asbahr FR, Manfro GG. Transtornos de ansiedade. Revista Brasileira de Psiquiatria 2000; 22(2,supl): 20-23. [citado em 20 de agosto de 2017]. Disponível em: http://www.scielo.br/scielo.php?script=sci_ arttext\&pid $=$ S151644462000000600006\&lang=pt.

10. Versiani M, Menezes GB, Fontenelle LF, Mululo S. Resistência ao Tratamento nos Transtornos de Ansiedade: Fobia Social, Transtorno de Ansiedade Generalizada e Transtorno do Pânico. Rev Brasileira de Psiquiatria 2007; 29(2,suppl): 55-60. [citado em 20 de agosto de 2017]. Disponível em: http://web.a.ebscohost.com/abstract?direct $=$ true\&profile=ehost $\&$ scope=site\&authtype $=$ crawler\&jrnl=15164446\&AN=27802373\& $\mathrm{h}$ =ICRsP0qtEB9kDucWcKT4Agh5xRmquE MZQhIK9zIKihpj929oc6gKST2L6qCdUSt\% 2f\%2bbTsJKt3U322zUS5Xg5vsw\%3d\%3d $\& c r l=c \& r e s u l t N s=A d m i n W e b A u t h \&$ resultLo cal=ErrCrlNotAuth\&crlhashurl=login. asp $\%$ 3fdirect\%3dtrue\%26profile\%3dehost\%26sc ope\%3dsite\%26authtype\%3dcrawler\%26jr nl\%3d15164446\%26AN\%3d27802373.

11. D'el Rey GJF, Greenberg PN, Husni MA, Cejkinski A. Terapia CognitivoComportamental de grupo no tratamento da fobia social generalizada. Rev Psicologia Argumento 2007; 25(50): 305-311. [citado em 15 setembro 2017]. Disponível em: https://periodicos.pucpr.br/index.php/psicol
ogiaargumento/article/viewFile/20551/1980 1.

12. Margis R, Kapcinski G. Transtorno de Ansiedade Generalizada. In: Knapp P, (org.). Terapia Cognitivo-Comportamental na Prática Psiquiátrica. Porto Alegre (RS): Artmed; 2004. p. 209-216.

13. Wright $\mathrm{JH}$, Basco $M$, Thase ME. Princípios Básicos da Terapia CognitivoComportamental. In: Wright JH, Basco M, Thase ME. Aprendendo a Terapia Cognitivo-Comportamental: Um Guia Ilustrado. Porto Alegre (RS): Artmed; 2008. p. 15-32.

14. Knapp P. Princípios Fundamentais da Terapia Cognitiva. In: Knapp P, (org.). Terapia Cognitivo-Comportamental na Prática Psiquiátrica. Porto Alegre (RS): Artmed; 2004. p. 19-41.

15. Sudak DM. Modelo e Teoria Cognitivos da Psicopatologia. In:

Terapia Cognitivo-Comportamental na Prática. Porto Alegre (RS): Artmed, 2008. p. 21-28.

16. Shinohara HO. Conceitualização da Terapia Cognitivo Comportamental. In: Banaco RA, (org.). Sobre Comportamento e Cognição: Aspectos Teóricos, Metodológicos e de Formação em Análise do Comportamento e Terapia Cognitivista. Santo André (SP): Esetec; 2001. p. 18-21.

17. Silva DS, Almeida RS, Braz ML, Crispim, MSDS, Acácio KPH, Nóbrega NKB. O uso dos Grupos Terapêuticos no Tratamento dos Transtornos de Ansiedade sob o Olhar da Terapia CognitivoComportamental. Rev Ciências Biológicas e da Saúde 2016; 3(3): 101-118. [citado em 15 de setembro de 2017]. Disponível em: https://periodicos.set.edu.br/index.php/fitsb iosaude/article/view/3395/2015.

18. Manfro GG, Heldt E, Shinohara $H$. Transtorno do Pânico. In: Knapp P, (org.). Terapia Cognitivo-Comportamental na Prática Psiquiátrica. Porto Alegre (RS): Artmed; 2004. p. 219-222. 
19. Moreno AL, Wainer R. Da Gnosiologia à Epistemologia: Um Caminho Científico para uma Terapia Baseada em Evidências. Rev Brasileira de Terapia Comportamental e Cognitiva 2014; 16(1): 41-54. [citado em 15 de setembro de 2017]. Disponível em: http://pepsic.bvsalud.org/pdf/rbtcc/v16n1/v 16n1a05.pdf.

20. Clark DA, Beck AT. Terapia Cognitiva para Transtornos de Ansiedade - Guia do Terapeuta. Porto Alegre: Artmed; 2012.

\section{Como citar (Vancouver)}

Moura IM, Rocha VHC, Bergamini GB, Samuelsson E, Joner C, Schneider LF et al. A terapia cognitivocomportamental no tratamento do transtorno de ansiedade generalizada. Rev Cient Fac Educ e Meio Ambiente [Internet]. 2018;9(1):423-441. DOI: http://dx.doi.org/10.31072/rcf.v9i1.557 